\title{
Relationship between peculiarities of atrial fibrillation, body mass index and adipokines levels
}

\author{
Oleksandr Bilovol, Yuliya Shaposhnikova, Iryna Ilchenko, Anna Shalimova \\ Kharkiv National Medical University, Department of Internal Medicine and Clinical Pharmacology, Kharkiv 61022, Ukraine.
}

Correspondence to: Dr. Anna Shalimova, Kharkiv National Medical University, Department of Internal Medicine and Clinical Pharmacology, Kharkiv 61022, Ukraine. E-mail: anna.shalimova83@gmail.com

How to cite this article: Bilovol O, Shaposhnikova Y, Ilchenko I, Shalimova A. Relationship between peculiarities of atrial fibrillation, body mass index and adipokines levels. Vessel Plus 2017;1:196-201.

\section{Article history: \\ Received: 8 Jun 2017 \\ Accepted: 11 Sep 2017 \\ Published: 28 Dec 2017}

\section{Key words:}

Atrial fibrillation,

obesity,

metabolically healthy obesity, metabolically unhealthy obesity, serum leptin,

high-molecular-weight adiponectin

\section{INTRODUCTION}

The likelihood of being overweight or obese in adulthood has been increasing in epidemic proportions in the last few decades ${ }^{[1]}$. We have witnessed a parallel rise in the incidence of atrial fibrillation (AF), which is the most common sustained cardiac arrhythmia and a significant cause of cardiovascular (CV) morbidity

\begin{abstract}
Aim: To assess the value of body mass index (BMI) and adipokine levels in predicting development of atrial fibrillation (AF) in the general population. Methods: Three hundred and ninety eight patients were examined for the presence of phenotype metabolically healthy obesity (MHO), according to the Wildman criteria; adipokine levels were assessed by enzyme immunoassay method; AF was assessed by electrocardiography (ECG) and/or by ECG diurnal monitoring. Results: Obesity (group 1) and overweight (group 2) was present in $23.7 \%$ and $42.0 \%$ of participants; while $21.1 \%$ were normal body weight (group 3) and $13.1 \%$ had a BMI $<19.9 \mathrm{~kg} / \mathrm{m}^{2}$ (group 4). Phenotype MHO was detected in 19.6\% patients. At follow-up, $32.4 \%$ of participants developed AF. Adiponectin levels were significantly higher in MHO patients as compared to metabolically unhealthy patients with abdominal obesity (AO). High molecular weight adiponectin (HMVAN) levels were significantly decreased in patients of groups 1 and 4 , as compared to groups 2 and 3. Correlation between AF and HMWAN was determined by regressive analysis in patients of 1 st and 4 th groups $(\beta=-0.24, P=0.003$ and $\beta=-0.26, P=$ 0.002 , respectively). Conclusion: The probability of developing AF increases with AO and decreased BMI, which is accompanied by a change in HMVAN levels. In MHO patients, the probability of AF developing is identical with persons having normal BMI.
\end{abstract}

and mortality. It can be explained by advances in the treatment of coronary heart disease (CHD) and heart failure (HF) improving life expectancy and consequently the prevalence of $A F$ (since the incidence of atrial fibrillation increases with age $)^{[2]}$.

Abdominal obesity (AO) and excess body weight are associated with the development of cardiovascular

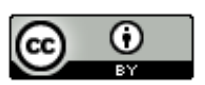

This is an open access article licensed under the terms of Creative Commons Attribution 4.0 International License (https://creativecommons.org/licenses/by/4.0/), which permits unrestricted use, distribution, and reproduction in any medium, as long as the original author is credited and the new creations are licensed under the identical terms.

For reprints contact: service@oaepublish.com

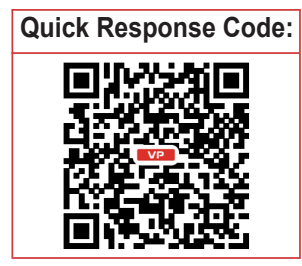


disease (CVD), including an increased risk of incidence of sudden cardiac death, atrium fibrillation and other form of arrhythmias ${ }^{[1]}$.

In contrast, a large review from Flegal et al. ${ }^{[3]}$ analyzed 97 studies of 2.9 million individuals, including > 270,000 deaths, and demonstrated that optimal survival occurred in "overweight" patients [body mass index (BMI): 25 to $30 \mathrm{~kg} / \mathrm{m}^{2}$ ], who had a significant $6 \%$ lower mortality than the "normal-weight" BMI cohort (BMI: 18.5 to $\left.25 \mathrm{~kg} / \mathrm{m}^{2}\right)^{[3]}$.

Patients with elevated BMI, butnormal insulin sensitivity, lipid profiles and blood pressure (BP), are considered to be metabolically healthy obesity $(\mathrm{MHO})^{[4]}$. Some epidemiological data suggest that $\mathrm{MHO}$ carries a lower risk of CVD, lower risk of AF development and less all-cause mortality than being normal weight yet metabolically unhealthy[ ${ }^{[5]}$. The precise mechanism that induces AF in obesity is still unknown. Metabolic comorbidities are common in obese people and could be the main reason for increased AF risk and total cardiovascular risk, apart from obesity itself. There are several proposed mechanisms that connect obesity and $A F^{[6-10]}$.

Enhanced neurohormonal activation, impaired insulin tolerance, dyslipoproteinemia, hypertension and pathological changes in circulating renin-angiotensinaldosterone system accompanies obesity and may contribute to left atrial enlargement and electrical instability, which may result in AF development ${ }^{[11]}$.

Studies on the mechanisms by which obesity induces AF show that obesity causes atrial arrhythmogenic remodeling. Progression of atrial fibrosis is a key event in the pathogenesis of $\mathrm{AF}^{[12,13]}$, and can be caused by aging, underlying cardiac diseases, systemic diseases or inflammatory processes, or AF itself. Obese individuals, even without cardiovascular disease, have left atrium (LA) enlargement ${ }^{[14]}$ and different electrical properties, such as slower conduction from the LA entering the pulmonary vein (PV) and a significantly shorter effective refractory period in the LA and $\mathrm{PV}^{[15]}$.

Adipokines are bioactive proteins produced by the adipose compartment that have wide-ranging effects across organs and tissues. Leptin and adiponectin are both closely related with obesity and have recently been implicated with AF. These hormones are secreted primarily by adipocytes, while the stromovascular tissue surrounding fat secretes tumor necrosis factor-alpha $(T N F-\alpha)$ and macrophages secrete resistin ${ }^{[16,17]}$. These adipokines have been proposed as links between adiposity and insulin resistance, glucose deregulation, and CVD. Adiponectin is reduced in obesity, CHD, HF, $\mathrm{AF}$ and T2DM, and increased following weight loss. Adiponectin levels were independently associated with the incident of $\mathrm{AF}^{[16,17]}$. However, it remains unclear whether these are markers of failed regulatory pathways that lead to AF or are directly involved in AF pathogenesis.

Our study aimed to elucidate the causal relationship between AF and BMI or adipokine levels, specifically serum leptin and high-molecular-weight adiponectin (HMWAN), among the general population.

\section{METHODS}

This study comprised 398 patients randomly selected while visiting the out-patient unit due to any reason, except acute heart pathology, provided they had not had heart rate disorders in their past medical history.

Among allotted patients there were 142 with arterial hypertension $(\mathrm{AH})(35.7 \%), 118$ suffering from ischemic heart disease (29.6\%), 73 with diabetes mellitus II type (DM-2) (18.3\%), provided DM-2 was compensated.

Also excluded were those patients who had developed kidney, liver diseases, heart diseases, heart failure (more than 2 functional class according to New York Heart Association), chronic obstructive lung pathology, malignant neoplasms or alcoholism.

Patients' age ranged from 37 to 56 years old (mean age: $41.4 \pm 2.3$ year). All patients involved in this study were examined according to the same protocol. Anthropometry was assessed with measurements of waist circumference and BMI calculated by Kettle formula. Additionally, BP was measured along with a panel of laboratory tests: fasting serum glucose (glucosoxidative approach); serum lipoproteins [serum cholesterol, triglycerides (TG), cholesterol of high-density lipoprotein (HDL) by enzyme colorimetric approach with kit of "Human" (Germany)]; C-reactive protein (CP) by solid phase enzyme immunoassay (EIA) (NycoCard CRP, Axis-Shield); serum Leptin by EIA kit Leptin ELISA [Diaghostics Biochem Canada Inc (DBC Inc) cat № ABIN362629 CAN-L-4260]; and serum HMWAN by EIA with use of kit DRG (USA). The homeostatic model assessment of insulin resistance (HOMA-IR) was calculated: $\mathrm{HOMA}-\mathrm{IR}=$ insulin, $\mathrm{mcU} / \mathrm{mL}$ $\times$ glucose, $\mathrm{mmol} / \mathrm{L} \div 22.5$ ) .

Overweight patients (BWl: $25-29.9 \mathrm{~kg} / \mathrm{m}^{2}$ ) and those with $\mathrm{AO}$ and $\mathrm{BMI} \geq 30 \mathrm{~kg} / \mathrm{m}^{2}$ were classified according to Wildman criteria (2008) as "MHO" and "metabolically 
unhealthy obesity (MUO)."

At the screening stage, echocardiography was performed on the apparatus Toshiba-SSH-60 A (Japan) using standard methods (in $\mathrm{M}$ and $\mathrm{B}$ regimens) by the recommendations of the American Echocardiogram Society.

The study included patients who initially had minimal differences in echocardiography indicators as compared to the control group for minimization of the impact of other risk factors at the onset of atrial fibrillation. Careful selection of patients made it possible to reliably estimate the effect of the selected parameters - BMI, leptin and high molecular weight adiponectin on the probability of atrial fibrillation, according to the goal of the study.

The phenotype $\mathrm{MHO}$ was assessed according to Wildman criteria: the presence of 0-1 factors indicating metabolic health ${ }^{[18]}$. Patients with $\mathrm{AO}$ were considered as $\mathrm{MHO}$ who had met the following criteria: systolic BP and diastolic BP below $130 \mathrm{mmHg}$ and $85 \mathrm{mmHg}$ respectively without antihypertensive therapy; serum TG $\leq 1.70 \mathrm{mmol} / \mathrm{L}$; serum HDL cholesterol $\geq 1.04 \mathrm{mmol} / \mathrm{L}$ for males and $\geq 1.30$ for females without lipid-reducing treatment; fasting serum glucose $\leq 5.55 \mathrm{mmol} / \mathrm{L}$ without hypoglycaemic therapy; serum $\mathrm{CP} \leq 4.72 \mathrm{mg} / \mathrm{L}$; HOMA$\mathrm{IR} \leq 4.81$.

Every patient was checked by electrocardiography (ECG) and/or by ECG diurnal monitoring (DM-ECG). Time of surveillance varied from 3.4 to 6.2 years (mean span $3.8 \pm 1.2$ years).

In order to evaluate obtained results they were compared with values of 20 people regarded as healthy (nothing abnormal was revealed) matching by age and gender. All statistical analyses were performed using the program (STATISTICA ${ }^{\circledR}$ for Windows 10.0) and $t$-criterion of Student $(P<0.05)$; the minimum level of statistical significance was assumed at $P<$ 0.05 . Continuous variables were presented as means \pm standard deviation. To determine the differences among the groups, Student's $t$-test was used for continuous variables, the correlation analysis was used to calculate the Pearson correlation coefficient and the Spearman rank correlation coefficient.

\section{RESULTS}

According to the goal of the study, all patients were allotted to 4 groups considering BMI [Table 1]. Echocardiographic characteristic of patients were given in Table 2. Characteristics of patients of different groups
Table 1: Subsets of patients considering BMI $(n=398)$

\begin{tabular}{lc}
\hline Groups of the patients $(\%)$ & BMI $\left(\mathbf{k g} / \mathbf{m}^{2}\right)$ \\
\hline Group 1 $(n=95)(23.9)$ & $\geq 30$ \\
Group 2 $(n=167)(42.0)$ & $25-29.9$ \\
Group 3 $(n=84)(21.1)$ & $20-24.9$ \\
Group 4 $(n=52)(13.0)$ & $<19.9$ \\
\hline
\end{tabular}

BMI: body mass index

included in the study, according to the main physical and laboratory parameters are presented in Table 3. Data for phenotype $\mathrm{MHO}$ assessment were accessible in 97 participants. Phenotype MHO (Wildman) was recognized in 19 people (19.6\%) that corresponded to the global estimates. Characteristics of MHO-type patients were given in Table 4. Assessment of serum adipokines revealed some peculiarities of leptin and HMWAN rate with regard to different BMI. HMWAN values were significantly raised in MHO-type patients comparatively to MUO type ones with $\mathrm{AO}$ : respectively $11.32 \mathrm{mcg} / \mathrm{mL}$ and $7.87 \mathrm{mcg} / \mathrm{mL}(P<0.01)$. Leptin was in the same range in patients of all subsets. HMWAN was significantly lower in patients of 1st and 4th groups comparatively to 2 nd and 3 rd groups [Table 5]. AF was identified in 129 patients (32.4\%) throughout the span of surveillance. Patients with obesity and body weight deficiency (1st and 4th groups) developed AF more frequently compared to other groups. There were different types of AF: AF paroxysms spontaneously resolved into sinus rhythm or restored to sinus rhythm by medicated cardioversion, long-lasting $A F$ of persistent or permanent type. Analysis of AF incidence among patients involved in the study was shown in Table 6.

Correlation between AF and HMWAN was determined by regressive analysis in patients of 1st and 4th groups $(\beta=-0.24, P=0.003$ and $\beta=-0.26, P=0.002$ respectively).

The probability of developing $A F$ increases with $A O$ and decreased $\mathrm{BMI}$, which is accompanied by a change in HMVAN levels. In MHO patients probability of AF developing is identical with normal BMI individuals.

\section{DISCUSSION}

The obtained results revealed some peculiarities of leptin and HMWAN levels in relation to various BMI. It was established that the level of leptin did not differ significantly in patients with normal body weight or with an increase or decrease BMI. At the same time, HMWAN value changes were more significant and had specific features in individuals with different body weight. We found a reduction of HMWAN with 
Table 2: Echocardiographic characteristic of patients

\begin{tabular}{lccccc}
\hline Indices & $\begin{array}{c}\text { Control } \\
(\boldsymbol{n}=\mathbf{2 0})\end{array}$ & $\begin{array}{c}\text { Group 1 } \\
(\boldsymbol{n}=\mathbf{9 5})\end{array}$ & $\begin{array}{c}\text { Group 2 } \\
(\boldsymbol{n}=\mathbf{1 6 7 )}\end{array}$ & $\begin{array}{c}\text { Group 3 } \\
(\boldsymbol{n}=\mathbf{8 4})\end{array}$ & $\begin{array}{c}\text { Group 4 } \\
(\boldsymbol{n}=\mathbf{5 2})\end{array}$ \\
\hline LA $(\mathrm{sm})$ & $2.9 \pm 0.2$ & $3.1 \pm 0.2$ & $3.3 \pm 0.4$ & $3.0 \pm 0.2$ & $2.6 \pm 0.3$ \\
EDV LV $(\mathrm{mL})$ & $98.5 \pm 4.3$ & $108.6 \pm 3.8$ & $110.8 \pm 4.2$ & $84.5 \pm 4.9$ & $96.6 \pm 3.8$ \\
ESV LV $(\mathrm{mL})$ & $38.4 \pm 2.2$ & $41.7 \pm 2.4$ & $42.8 \pm 3.6$ & $37.0 \pm 3.1$ & $38.6 \pm 3.8$ \\
EF $(\%)$ & $64.4 \pm 4.2$ & $62.8 \pm 3.8$ & $57.2 \pm 4.2$ & $59.6 \pm 5.3$ & $61.6 \pm 3.8$ \\
TPWd (mm) & $8.4 \pm 0.4$ & $9.2 \pm 1.2$ & $9.0 \pm 1.4$ & $8.6 \pm 1.1$ & $8.2 \pm 0.8$ \\
TIVSd (mm) & $8.2 \pm 0.6$ & $9.2 \pm 1.8$ & $9.1 \pm 1.2$ & $8.5 \pm 1.2$ & $8.2 \pm 0.8$ \\
\hline
\end{tabular}

LA: left atrium; EDV LV: end-diastolic volume of the left ventricle; ESV LV: end-systolic volume of the left ventricle; EF: ejection fraction; TPWd: thickness of the posterior wall of the left ventricle in diastole; TIVSd: the thickness of the interventricular septum in diastole

Table 3: Baseline characteristics of the study population with different BMI

\begin{tabular}{|c|c|c|c|c|c|}
\hline \multirow[b]{2}{*}{ Index } & \multirow{2}{*}{$\begin{array}{l}\text { Control group } \\
\qquad(n=20)\end{array}$} & \multicolumn{4}{|c|}{ Examined patients $(n=398)$} \\
\hline & & $\begin{array}{l}\text { Group } 1 \\
(n=95)\end{array}$ & $\begin{array}{l}\text { Group } 2 \\
(n=167)\end{array}$ & $\begin{array}{l}\text { Group } 3 \\
(n=84)\end{array}$ & $\begin{array}{c}\text { Group } 4 \\
(n=52)\end{array}$ \\
\hline WC (sm) & $78.2 \pm 5.4$ & $101.4 \pm 6.2^{* * *}$ & $96.8 \pm 4.4^{* * *}$ & $82.6 \pm 6.4^{\wedge, \#, * *}$ & $64.8 \pm 2.6^{\wedge, \#}$ \\
\hline $\mathrm{HC}(\mathrm{sm})$ & $92.4 \pm 6.3$ & $142.8 \pm 8.4^{*, 8, *}$ & $124.6 \pm 7.4$ & $96.5 \pm 6.51^{\wedge, \#, *}$ & $80.2 \pm 3.4^{\wedge, \#}$ \\
\hline $\mathrm{WC} / \mathrm{HC}$ & $0.76 \pm 0.04$ & $0.82 \pm 0.08$ & $0.80 \pm 0.07$ & $0.80 \pm 0.06$ & $0.78 \pm 0.06$ \\
\hline $\mathrm{SAD}(\mathrm{mmHg})$ & $120.4 \pm 2.6$ & $142.4 \pm 6.8^{* * * *}$ & $138.8 \pm 5.2^{* * *}$ & $132.5 \pm 3.5^{\wedge * *}$ & $118.8 \pm 4.6$ \\
\hline $\mathrm{DAD}(\mathrm{mmHg})$ & $78.4 \pm 2.2$ & $88.4 \pm 4.5^{*, * *}$ & $86,2 \pm 3.4^{*, *}$ & $82.4 \pm 6.2^{\wedge * *}$ & $74.6 \pm 3.8^{\wedge, \#}$ \\
\hline Total cholesterol (mmol/L) & $4.42 \pm 0.08$ & $6.68 \pm 1.07^{* * *}$ & $6.24 \pm 1.02^{* * *}$ & $5.16 \pm 1.29$ & $4.14 \pm 0.08^{\wedge, \#}$ \\
\hline Triglycerides (mmol/L) & $1.34 \pm 0.06$ & $3.22 \pm 0.08$ & $3.12 \pm 0.06$ & $2.16 \pm 0.07^{\wedge, \#, * *}$ & $1.04 \pm 0.03^{\wedge, \#}$ \\
\hline HDL cholesterol (mmol/L) & $1.40 \pm 0.04$ & $0.72 \pm 0.06 \%$ & $0.84 \pm 0.04$ & $1.05 \pm 0.02^{\wedge, \#}$ & $1.02 \pm 0.02^{\wedge}, \#$ \\
\hline Fasting glucose (mmol/L) & $4.60 \pm 0.22$ & $6.28 \pm 0.36^{* * *}$ & $6.02 \pm 0.16^{* * *}$ & $5.12 \pm 0.08^{\wedge, \#, * *}$ & $3.72 \pm 0.18^{\wedge, \#}$ \\
\hline HOMA-IR & $1.88 \pm 0.22$ & $5.12 \pm 0.32^{*, \&, *}$ & $4.98 \pm 0.28^{*, \alpha^{* *}}$ & $2.32 \pm 0.24^{\wedge, \#}$ & $1.14 \pm 0.06^{\wedge, \#}$ \\
\hline C-reactive protein (mg/L) & $2.24 \pm 0.12$ & $5.38 \pm 1.24$ & $4.42 \pm 1.12$ & $3.02 \pm 0.79^{\wedge}$ & $2.68 \pm 0.32^{\wedge, \#}$ \\
\hline Serum leptin $(\mathrm{mcg} / \mathrm{mL})$ & $21.6 \pm 1.2$ & $22.5 \pm 1.6$ & $23.5 \pm 1.8$ & $22.4 \pm 1.4$ & $21.9 \pm 1.2$ \\
\hline HMWAN (mcg/mL) & $14.74 \pm 1.22$ & $4.42 \pm 1.06^{*, \#, 8}$ & $13.82 \pm 1.12^{\wedge . * *}$ & $14.23 \pm 1.07^{1 * *}$ & $3.75 \pm 0.18^{*, \#, \&}$ \\
\hline
\end{tabular}

*: reliability of differences $(P \leq 0.05)$ compared with control group; $\wedge$ : reliability of differences $(P \leq 0.05)$ compared with group 1 ; \#: reliability of differences $(P<0.05)$ compared with group 2; \&: reliability of differences $(P<0.05)$ compared with group 3 ; **: reliability of differences $(P<0.05)$ compared with group 4; WC: waist circumference; HC: hip circumference; SAD: systolic blood pressure; DAD: diastolic blood pressure; HDL: high-density lipoprotein; HOMA-IR: homeostasis model of assessment insulin resistance; BMI: body mass index

BMI deviations greater and smaller from normal values. Thus, there was significant decrease of serum HMWAN in patients with obesity of group 1 with a BMI of more than $30 \mathrm{~kg} / \mathrm{m}^{2}$, and with a body mass deficit in patients of group 4 with BMI less than $19.9 \mathrm{~kg} / \mathrm{m}^{2}$. It is also important to note that the difference in the HMWAN value reduction in patients with obesity and body weight deficit was insignificant, while significantly different from those with normal and increased body weight with a BMI within $25-29.9 \mathrm{~kg} / \mathrm{m}^{2}$.

During the observation period, AF most often developed in patients who had the most significant initial HMWAN reduction - in patients with more prominent obesity and in patients with body mass deficiency in groups
1 and 4. Less often, AF developed in individuals with increased body weight, even without obesity and only in isolated cases in patients with normal body weight. The most frequent variant of $A F$ was paroxysmal $A F$, while the other variants - persistent or permanent type, developed much less frequently.

These changes have been confirmed by established correlation dependence of the development of $\mathrm{AF}$ and HMWAN reduction in groups 1 and 4 of the patients. It was found that significant BMI deviation in both directions is a prognostically unfavorable factor for the development of AF in patients, even without any other manifestations of CV disease or metabolic abnormality. It was found that the deviation of BMI 
Table 4: Characteristics of MHO-type patients by Wildman criteria

\begin{tabular}{lccc}
\hline Index & Wildman criteria & MHO-type patients & $P$ \\
SAD $(\mathrm{mmHg})$ & $\leq 130$ & $123.4 \pm 2.4$ & 0.05 \\
DAD $(\mathrm{mmHg})$ & $\leq 85$ & $78.8 \pm 2.2$ & 0.05 \\
Triglycerides (mmol/L) & $\leq 1.70$ & $1.46 \pm 0.18$ & 0.05 \\
HDL cholesterol: men, & $\geq 1.04$ & $1.15 \pm 0.08$ & 0.05 \\
women $(\mathrm{mmol} / \mathrm{L})$ & $\geq 1.30$ & $1.42 \pm 0.11$ & 0.05 \\
Fasting blood glucose (mmol/L) & $\leq 5.55$ & $4.08 \pm 0.06$ & 0.05 \\
C-reactive protein (mg/L) & $<4.72$ & $2.18 \pm 0.06$ & 0.05 \\
HOMA-IR & $<4.81$ & $2.62 \pm 0.12$ & 0.05 \\
\hline
\end{tabular}

MHO: metabolically healthy obesity; SAD: systolic blood pressure; DAD: diastolic blood pressure; HDL: high-density lipoprotein; HOMA-IR: homeostasis model of assessment insulin resistance

Table 5: Serum leptin and high-molecular-weight adiponectin values in patients with different body weight

\begin{tabular}{lccccc}
\hline & Control group & \multicolumn{4}{c}{ Examined patients $(\boldsymbol{n}=\mathbf{3 9 8})$} \\
\cline { 3 - 6 } Index & $(\boldsymbol{n}=\mathbf{2 0})$ & Group 1 $(\boldsymbol{n}=\mathbf{9 5})$ & Group 2 $(\boldsymbol{n}=\mathbf{1 6 7})$ & Group 3 $(\boldsymbol{n}=\mathbf{8 4})$ & Group 4 $(\boldsymbol{n}=\mathbf{5 2})$ \\
\hline Serum leptin $(\mathrm{mcg} / \mathrm{mL})$ & $21.6 \pm 1.2$ & $22.5 \pm 1.6$ & $23.5 \pm 1.8$ & $22.4 \pm 1.4$ & $21.9 \pm 1.2$ \\
HMWAN $(\mathrm{mcg} / \mathrm{mL})$ & $14.74 \pm 1.22$ & $4.42 \pm 1.06^{*, \#, \&}$ & $13.82 \pm 1.12^{\&, *}$ & $14.23 \pm 1.07^{\&, * *}$ & $3.75 \pm 0.18^{*, \#, \&}$ \\
\hline
\end{tabular}

*: reliability of differences $(P \leq 0.05)$ compared with control group; $\wedge$ : reliability of differences $(P \leq 0.05)$ compared with group 1 ; \#: reliability of differences $(P<0.05)$ compared with group 2; \&: reliability of differences $(P<0.05)$ compared with group 3 ; ${ }^{* *}$ : reliability of differences $(P<0.05)$ compared with group 4

Table 6: The incidence of various forms of atrial fibrillation in patients examined during the observation period (3.8 \pm 1.2 years)

\begin{tabular}{lcccc}
\hline \multirow{2}{*}{ Index } & \multicolumn{4}{c}{ Examined patients $(\boldsymbol{n}=\mathbf{3 9 8})(\%)$} \\
\cline { 2 - 5 } & Group 1 $(\boldsymbol{n}=\mathbf{9 5})$ & Group 2 $(\boldsymbol{n}=\mathbf{1 6 7 )}$ & Group 3 $(\boldsymbol{n}=\mathbf{8 4})$ & Group 4 ( $\boldsymbol{n}=\mathbf{5 2})$ \\
\hline Paroxysmal atrial fibrillation (AFpx) & $24(25.3)$ & $26(15.6)$ & $7(8.3)$ & $13(25.0)$ \\
Persistent atrial fibrillation (AFps) & $17(17.9)$ & $24(14.4)$ & $4(4.8)$ & $9(17.3)$ \\
Permanent atrial fibrillation (AFpm) & $11(11.6)$ & $19(11.4)$ & $2(2.4)$ & $7(13.5)$ \\
\hline
\end{tabular}

both in the direction of increase and decrease is a prognostically unfavorable factor for the development of $A F$ in patients even without any other manifestations of $\mathrm{CV}$ disease.

These results indicate that obesity not only leads to different metabolic, hormonal and hemodynamic changes in the body that affect the heart muscle, causing its structural and functional changes, but the same changes are taking place in the patient with a body weight deficit, causing similar pathogenesis, particularly AF development.

Adiponectin plays an important role in many metabolic processes; it has a protective effect, especially for development of endothelial dysfunction, atherosclerosis and other vascular diseases, the progression of which is reflected in heart rhythm disturbances, in particular, the onset of $\mathrm{AF}^{[19]}$. Adiponectin is under the influence and itself influences the action of many pathophysiological mechanisms, including pathological TNF- $\alpha$, interleukin-6, C-reactive protein, insulin, body weight, blood pressure deviation, and progression of many chronic diseases ${ }^{[20]}$.
Adiponectin is involved in cardiac remodeling, not only through direct action on the cardiac muscle, but indirectly through the effect on endothelial function, atherogenesis, and vascular inflammation. In addition, adiponectin penetrates the blood-brain barrier and affects the function of the heart through the central nervous system ${ }^{[21]}$.

Adipose tissue can be related to the lipotoxicity of cardiomyocytes, which is carried out through unknown factors with the participation of FATP4 and CD36 transporters, and cardiomyocytes can maintain the stability of adipose tissue, affecting the secretion of adipokines $^{[22]}$.

Insulin resistance - this is the main pathological mechanism that binds metabolic, anthropometric and clinical indicators with the increasing risk of CVD and DM2. Adipocytokine imbalance, with a low level of adiponectin, can act as a triggering mechanism for the development of hyperinsulinemia, impaired glucose tolerance, dyslipidemia, endothelial dysfunction, arterial hypertension, abdominal obesity, fibrosis and atrial fibrillation ${ }^{[23]}$. 


\section{DECLARATIONS}

\section{Authors' contributions}

Study design: O. Bilovol, I. Ilchenko

Development of methodology: O. Bilovol

Collection of data: O. Bilovol, Y. Shaposhnikova, I. Ilchenko, A. Shalimova

Analysis and/or interpretation of data: O. Bilovol, Y. Shaposhnikova, I. Ilchenko, A. Shalimova

Writing (not revising) all or sections of the manuscript: O. Bilovol, Y. Shaposhnikova, I. Ilchenko, A. Shalimova Manuscript review: O. Bilovol, I. Ilchenko

Supervision: O. Bilovol

\section{Financial support and sponsorship None.}

\section{Conflicts of interest}

There are no conflicts of interest.

\section{Patient consent}

Each patient was informed the study and gave their consent.

\section{Ethics approval}

The study protocol was supported by the Ethics Committee of the Kharkiv National Medical University.

\section{REFERENCES}

1. Ortega FB, Lavie CJ, Blair SN. Obesity and cardiovascular disease. Circ Res 2016;118:1752-70.

2. Asghar O, Alam U, Hayat SA, Aghamohammadzadeh R, Heagerty AM, Malik RA. Obesity, diabetes and atrial fibrillation; epidemiology, mechanisms and interventions. Curr Cardiol Rev 2012;8:253-64.

3. Flegal KM, Kit BK, Orpana H, Graubard BI. Association of allcause mortality with overweight and obesity using standard body mass index categories: a systematic review and meta-analysis. JAMA 2013;309:71-82

4. Lee H, Choi EK, Lee SH, Han KD, Rhee TM, Park CS, Lee SR, Choe WS, Lim WH, Kang SH, Cha MJ, Oh S. Atrial fibrillation risk in metabolically healthy obesity: a nationwide population-based study. Int J Cardiol 2017;240:221-7.

5. Payvar S, Kim S, Rao SV, Krone R, Neely M, Paladugu N, Daggubati R. In-hospital outcomes of percutaneous coronary interventions in extremely obese and normal-weight patients: findings from the NCDR (National Cardiovascular Data Registry). J Am Coll Cardiol 2013;62:692-6.

6. Wanahita N, Messerli FH, Bangalore S, Gami AS, Somers VK, Steinberg JS. Atrial fibrillation and obesity--results of a meta-analysis. Am Heart J 2008;155:310-5.

7. Wang TJ, Parise H, Levy D, D'Agostino RB Sr, Wolf PA, Vasan RS, Benjamin EJ. Obesity and the risk of new-onset atrial fibrillation.
JAMA 2004;292:2471-7.

8. Guglin M, Maradia K, Chen R, Curtis AB. Relation of obesity to recurrence rate and burden of atrial fibrillation. Am J Cardiol 2011;107:579-82.

9. Frost L, Hune LJ, Vestergaard P. Overweight and obesity as risk factors for atrial fibrillation or flutter: the Danish diet, cancer, and health study. Am J Med 2005;118:489-95.

10. Murphy NF, MacIntyre K, Stewart S, Hart CL, Hole D, McMurray JJ. Long-term cardiovascular consequences of obesity: 20-year followup of more than 15000 middle-aged men and women (the RenfrewPaisley study). Eur Heart $J$ 2006;27:96-106.

11. Goette A, Staack T, Röcken C, Arndt M, Geller JC, Huth C, Ansorge $\mathrm{S}$, Klein HU, Lendeckel U. Increased expression of extracellular signal-regulated kinase and angiotensin-converting enzyme in human atria during atrial fibrillation. J Am Coll Cardiol 2000;35:1669-77.

12. Dzeshka MS, Lip GY, Snezhitskiy V, Shantsila E. Cardiac fibrosis in patients with atrial fibrillation: mechanisms and clinical implications. J Am Coll Cardiol 2015;66:943-59.

13. Nattel S, Harada M. Atrial remodeling and atrial fibrillation: recent advances and translational perspectives. $\mathrm{J} \mathrm{Am} \mathrm{Coll} \mathrm{Cardiol}$ 2014;63:2335-45

14. Ito K, Date T, Kawai M, Nojiri A, Narui R, Hioki M, Tanigawa S, Yamashita S, Tokuda M, Inada K, Matsuo S, Yamane T, Yoshimura M. Morphological change of left atrium in obese individuals. Int $J$ Cardiol 2011;152:117-9.

15. Munger TM, Dong YX, Masaki M, Oh JK, Mankad SV, Borlaug BA Asirvatham SJ, Shen WK, Lee HC, Bielinski SJ, Hodge DO, Herges RM, Buescher TL, Wu JH, Ma C, Zhang Y, Chen PS, Packer DL, Cha YM. Electrophysiological and hemodynamic characteristics associated with obesity in patients with atrial fibrillation. $J \mathrm{Am}$ Coll Cardiol 2012;60:851-60.

16. Ouchi N, Parker JL, Lugus JJ, Walsh K. Adipokines in inflammation and metabolic disease. Nat Rev Immunol 2011;11:85-97.

17. Van de Voorde J, Pauwels B, Boydens C, Decaluwé K. Adipocytokines in relation to cardiovascular disease. Metabolism 2013;62:1513-21.

18. Wildman RP, Muntner P, Reynolds K, McGinn AP, Rajpathak S, Wylie-Rosett J, Sowers MR. The obese without cardiometabolic risk factor clustering and the normal weight with cardiometabolic risk factor clustering: prevalence and correlates of 2 phenotypes among the US population (NHANES 1999-2004). Arch Intern Med 2008;168:1617-24.

19. Macheret F, Bartz TM, Djousse L, Ix JH, Mukamal KJ, Zieman SJ, Siscovick DS, Tracy RP, Heckbert SR, Psaty BM, Kizer JR. Higher circulating adiponectin levels are associated with increased risk of atrial fibrillation in older adults. Heart 2015;101:1368-74.

20. Jung UJ, Choi MS. Obesity and its metabolic complications: the role of adipokines and the relationship between obesity, inflammation, insulin resistance, dyslipidemia and nonalcoholic fatty liver disease. Int J Mol Sci 2014;15:6184-223.

21. Kizer JR, Benkeser D, Arnold AM, Djousse L, Zieman SJ, Mukama KJ, Tracy RP, Mantzoros CS, Siscovick DS, Gottdiener JS, Ix JH. Total and high-molecular-weight adiponectin and risk of coronary heart disease and ischemic stroke in older adults. J Clin Endocrinol Metab 2013;98:255-63.

22. Anan M, Uchihashi K, Aoki S, Matsunobu A, Ootani A, Node K, Toda $\mathrm{S}$. A promising culture model for analyzing the interaction between adipose tissue and cardiomyocytes. Endocrinology 2011;152:1599-605.

23. Colwell JA. Type 2 diabetes, pre-diabetes, and the metabolic syndrome. JAMA 2011;306:215. 